Research Paper

\title{
Chemoradiotherapy Versus Radiotherapy Alone in Stage II Nasopharyngeal Carcinoma: A Systemic Review and Meta-analysis of 2138 Patients
}

\author{
Cheng $\mathrm{Xu}^{1 *}$, Li-He Zhang ${ }^{1 *}$, Yu-Pei Chen ${ }^{1^{*}}, \mathrm{Xu} \mathrm{Liu}^{1}$, Guan-Qun Zhou1 ${ }^{1}$ Ai-Hua Lin², Ying Sun ${ }^{1}$, Jun Ma1凶 \\ 1. Department of Radiation Oncology, Sun Yat-sen University Cancer Center, State Key Laboratory of Oncology in South China, Collaborative Innovation \\ Center of Cancer Medicine, Guangzhou, People's Republic of China; \\ 2. Department of Medical Statistics and Epidemiology, School of Public Health, Sun Yat-sen University, Guangzhou, People's Republic of China. \\ * Cheng Xu, Li-He Zhang, and Yu-Pei Chen contributed equally to this work.
}

$\square$ Corresponding author: Jun Ma, Department of Radiation Oncology, Sun Yat-sen University Cancer Center, State Key Laboratory of Oncology in South China, Collaborative Innovation Center of Cancer Medicine, 651 Dongfeng Road East, Guangzhou 510060, People's Republic of China. Tel.: +86-20-87343469; Fax: +86-20-87343295; E-mail: majun2@mail.sysu.edu.cn.

(c) Ivyspring International Publisher. This is an open access article distributed under the terms of the Creative Commons Attribution (CC BY-NC) license (https://creativecommons.org/licenses/by-nc/4.0/). See http://ivyspring.com/terms for full terms and conditions.

Received: 2016.08.23; Accepted: 2016.10.13; Published: 2017.01.15

\begin{abstract}
Background: To explore the value of chemoradiotherapy (CRT) in stage II nasopharyngeal carcinoma (NPC) compared to radiotherapy (RT) alone which includes two-dimensional radiotherapy (2D-RT) and intensity-modulated radiotherapy (IMRT).

Methods: All topic-related comparative articles were identified by a comprehensive search of public databases (MEDLINE, EMBASE, Cochrane Library and CBMdisc). The primary outcomes were overall survival (OS), loco-regional relapse-free survival (LRRFS) and distant metastasis-free survival (DMFS). Secondary outcomes were grade 3-4 acute toxicity events. We performed subgroup analysis of CRT versus $2 D-R T / I M R T$ alone to investigate the optimal modality. Sensitivity analysis focused on CRT versus IMRT alone was used to assess stability of the study results.

Results: Eleven comparative studies (2138 patients) were eligible. CRT had significantly higher OS $(\mathrm{HR}=0.67,95 \% \mathrm{Cl}=0.45-0.98, P=0.04)$ and LRRFS $(\mathrm{HR}=0.61,95 \% \mathrm{Cl}=0.46-0.80, P=0.0003)$ than RT alone, but no significant difference was observed in DMFS $(\mathrm{HR}=0.83,95 \% \mathrm{Cl}=0.52-1.31$, $P=0.41$ ). Meanwhile, CRT was associated with higher frequencies of grade 3-4 leukopenia, mucositis and nausea $(P=0.005,0.03,<0.0001$, respectively). Subgroup analysis showed that IMRT alone could achieve equivalent OS, LRRFS and DMFS compared to CRT $(P=0.14,0.06,0.89$, respectively). Significant value was only observed in LRRFS for CRT compared to $2 \mathrm{D}-\mathrm{RT}$ alone ( $P$ $=0.01$ ). Sensitivity analysis for the comparison of CRT and IMRT alone demonstrated generally stable outcomes, in support of the final conclusions.

Conclusions: In the treatment of patients with stage II NPC, CRT was better than 2D-RT alone with significant benefit in LRRFS. IMRT alone was superior to CRT with equivalent survival outcomes and fewer grade 3-4 acute toxicities.
\end{abstract}

Key words: nasopharyngeal carcinoma; stage II; chemoradiotherapy; radiotherapy; intensity-modulated radiotherapy; meta-analysis.

\section{Introduction}

Nasopharyngeal carcinoma (NPC) is a malignant head and neck cancer, which has a relatively high incidence of 20-30 per 100000 in endemic areas such as southern China and Southeast Asia [1]. NPC is both radiosensitive and chemosensitive; radiotherapy (RT) and various chemoradiotherapy (CRT) schedules are widely used in clinical practice. Concurrent chemoradiotherapy (CCRT) with or without adjuvant 
chemotherapy $(A C)$ is regarded as the first choice for loco-regionally advanced NPC (stage III-IVb), and RT alone is the recommended strategy for stage I NPC [2, 3]. However, there are still a lot of controversies in the treatment of patients with stage II NPC.

Only one randomized controlled trial (RCT) compared CRT with two-dimensional radiotherapy (2D-RT) alone in stage II NPC [4]. This study reported that CRT could significantly improve 5-year overall survival (OS) and distant metastasis-free survival (DMFS), but not loco-regional relapse-free survival (LRRFS). However, data from several retrospective studies comparing CRT with 2D-RT alone in stage II NPC showed conflicting results, with non-significant benefit in all survival outcomes [5] or significant benefit only in LRRFS favoring CRT [6]. Importantly, an individual patient data meta-analysis reported that the addition of chemotherapy should be implemented for loco-regionally advanced NPC but not early stage disease [7].

After the advent of intensity-modulated radiotherapy (IMRT), its therapeutic performance has been demonstrated more efficient than conventional $\mathrm{RT}$, since it provides an adequate dose to the primary tumor while enabling improved sparing of the surrounding normal tissues $[8,9]$. It has been reported that patients with early stage NPC treated with IMRT alone can achieve 5-year LRRFS and DMFS of 97.7\% and $97.8 \%$, and 3-year OS of $96.2 \%$ with tolerable toxicities [10, 11]. However, retrospective studies which compared CRT with IMRT alone in stage II NPC showed inconsistent survival outcomes [12-17]; topic-related RCTs were absent from publication. As such, the benefit of CRT in stage II NPC still remains unclear.

In attempt to address this question, we undertook the first meta-analysis of all topic-related comparative studies to explore the value of CRT compared to RT alone in stage II NPC.

\section{Materials and methods}

A prospective protocol was initially planned according to the Preferred Reporting Items for Systematic Reviews and Meta-Analysis (PRISMA) and Meta-analysis Of Observational Studies in Epidemiology recommendations for study reporting (MOOSE) $[18,19]$.

\section{Identification and eligibility of relevant studies}

A comprehensive literature search was performed using MEDLINE, EMBASE, Cochrane Library and the CBMdisc database for Chinese articles without restrictions to language or region (last updated on June 29, 2016). The following MeSH terms and their combinations were searched in
[Title/Abstract]: stage II/early stage, nasopharyngeal carcinoma/nasopharyngeal cancer/nasopharyngeal neoplasm/NPC. After the initial screening of the title and abstract of retrieved literatures, the full text of relevant articles was independently assessed by two investigators for inclusion (C.X. and L.H.Z.) and any disagreements were resolved by consensus. The related article function and manual searches of reference lists were also carried out to expand the included studies.

The studies included in this meta-analysis complied with all of the following predefined criteria: (1) studies that compared CRT (i.e., CCRT, AC, induction chemotherapy $[\mathrm{IC}]$ and their combinations) with 2D-RT/IMRT alone in stage II NPC were included; (2) patients had newly-diagnosed, pathologically-confirmed NPC without previous treatment; (3) at least one of the following outcomes could be extracted directly from the contents of the paper or indirectly by Tierney's Methods: time-to-event data such as OS, LRRFS and DMFS, occurrence of grade 3-4 acute toxicity events [20]; (4) when multiple articles from the same institution were published, articles that examined different populations during non-overlapping time intervals or studies by different authors were included; (5) editorials, letters to editors, reviews, case reports, basic research reports and conference abstracts were excluded.

\section{Data extraction}

Two investigators (C.X. and L.H.Z.) independently extracted and summarized the data from all included studies using a standardized data extraction form. For each study, the following items were extracted: first author, year of publication, country of origin, inclusion period, demographic data, study design, median follow-up time and range, staging system (e.g., the 1997/2002/2010 edition of the American Joint Committee on Cancer [AJCC] staging system) and detailed staging data, World Health Organization (WHO) histologic type, description of radiotherapy parameters and chemotherapy regimen, time-to-event data (OS, LRRFS, DMFS) and occurrence of grade 3-4 acute toxicity events.

The primary outcomes were OS, LRRFS and DMFS. The definition of OS was the time from distribution until death due to any cause or the latest date known to be alive. LRRFS and DMFS were defined as the duration from the date of initiating treatment to local/regional relapse and distant metastasis, respectively. The secondary outcomes were the rates of grade 3-4 acute toxicities, including hematological events (leukopenia, anemia and 
thrombocytopenia) and non-hematological events (liver dysfunction, renal impairment, nausea, mucositis and dermatitis).

\section{Quality assessment and data analysis}

The methodological quality of RCTs was assessed by the Cochrane risk of bias tool [21]; the methodological quality of retrospective studies was appraised using the modified Newcastle-Ottawa scale, which comprises three items: patient selection, comparability of the study groups, and assessment of outcomes [22]. The quality of each retrospective study was scored on a scale ranging from 0 to 9 by two independent investigators (C.X. and L.H.Z.). Studies with scores $\geq 6$ were regarded as high-quality. The level of evidence for each study was assessed according to the criteria published by the Centre for Evidence-Based Medline in Oxford, UK [23].

To evaluate the agreement between the investigators regarding their assessment of study quality, we calculated the kappa coefficient for inter-rater reliability; $P$-value $<0.05$ indicated a good agreement. Hazard ratios (HRs) and 95\% confidence intervals (CIs) were used as summary statistics for time-to-event data. The natural logarithm of HR $(\operatorname{lnHR})$ and standard error of the $\operatorname{lnHR}(\operatorname{se}(\operatorname{lnHR}))$ were calculated to figure out pooled HRs and 95\% CIs for meta-analysis according to the guidelines by Parmar [24]. For dichotomous variables, the risk ratios (RRs) and 95\% CIs were used as summary statistics. A HR or RR $<1$ represented a survival or safety benefit favoring the CRT group. If the $95 \% \mathrm{CI}$ was $<1$, the benefit of CRT was statistically significant $(P<0.05)$. Statistical heterogeneity between studies was appraised using the Chi-square $\left(X^{2}\right)$ and I-square $\left(I^{2}\right)$ test, with significance set at $P<0.10$. Moreover, $I^{2}$-value $>25 \%, 50 \%$, or $75 \%$ was considered to have low, moderate, or high heterogeneity [25]. If significant heterogeneity existed, the random-effects model was used; otherwise, the fixed-effects model was used.

Subgroup analyses of CRT versus 2D-RT alone and CRT versus IMRT alone were performed to explore the optimal modality of treatment. Sensitivity analysis of CRT versus IMRT alone was carried out to assess stability of the study results. It was performed by analyzing the results after sequential exclusion of some studies from the meta-analysis according to different matching criteria. Funnel plots and Begg's tests were performed to detect potential publication bias using Stata software 12.0 (StataCorp, College Station, TX, USA) [26]. The meta-analysis and forest plots were produced by Review Manager 5.3
(Cochrane Collaboration, Oxford, UK).

\section{Results}

\section{Study identification and description}

After the rounds of selection presented in Figure 1, eleven studies assessing 2138 patients (1237 patients treated with CRT and 901 patients treated with RT alone) fulfilled the predefined inclusion criteria and were included in this meta-analysis [4-6, 12-17, 27, 28].

Table 1 presents the baseline characteristics of the eligible studies. Ten of the eleven included articles were retrospective studies [5, 6, 12-17, 27, 28]; the remaining one was an RCT [4]. All studies were performed in Asia, of which, ten studies were published in English [4-6, 12-17, 28], and one was in Chinese with English abstract [27]. All studies recruited patients with stage II NPC; however, two studies also individually included a small proportion of patients with stage I (24.6\%: T1N0M0) [13] and stage III (21.4\%: T3NOM0) disease [16]. The summary of radiotherapy parameters and chemotherapy regimens of the included studies is shown in Table 2 . Seven studies compared CRT with IMRT alone in all or most patients with stage II NPC [12-17, 27]. Four studies compared CRT with 2D-RT alone [4-6, 28].

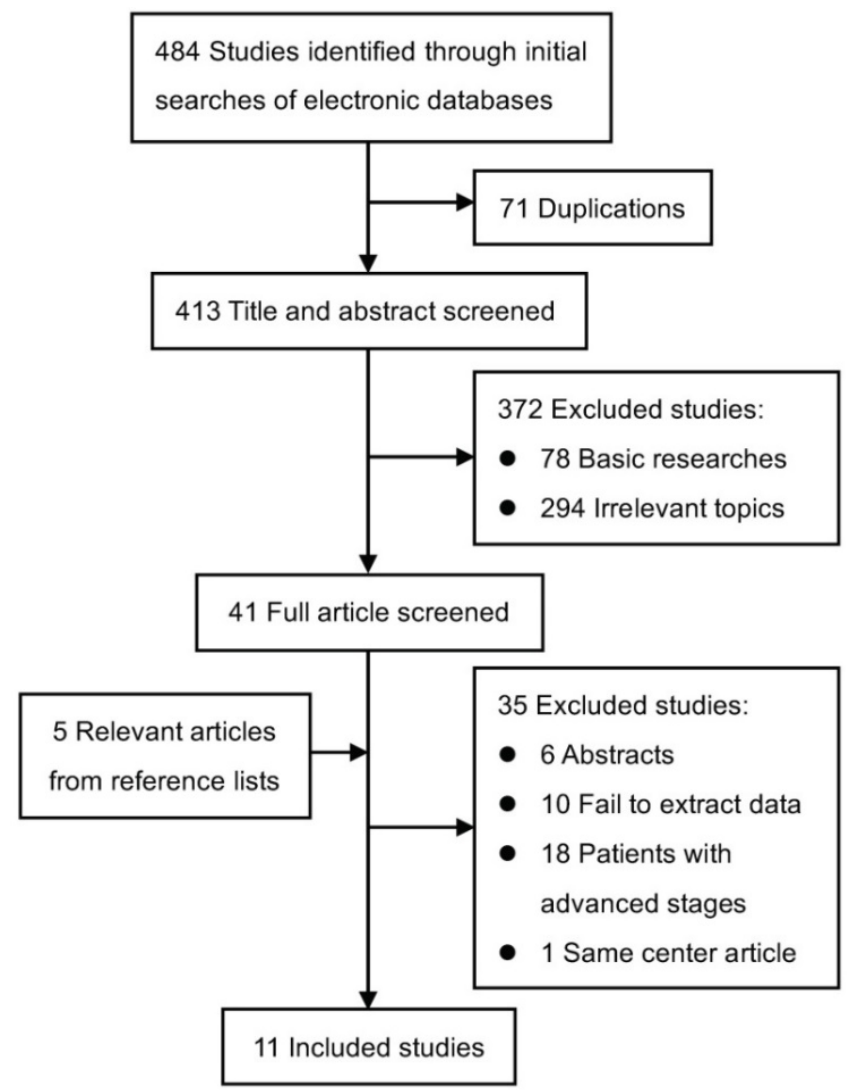

Figure 1. Flow diagram of study identification, exclusion and inclusion. 
Table 1. Baseline characteristics of included studies

\begin{tabular}{|c|c|c|c|c|c|c|c|c|c|c|c|c|}
\hline $\begin{array}{l}\text { First author/ } \\
\text { year }\end{array}$ & $\begin{array}{l}\text { Country } \\
\text { of origin }\end{array}$ & $\begin{array}{l}\text { Study } \\
\text { language }\end{array}$ & $\begin{array}{l}\text { Time } \\
\text { range }\end{array}$ & $\begin{array}{l}\text { Study } \\
\text { design }\end{array}$ & $\begin{array}{l}\text { No. of } \\
\text { patients } \\
(\mathrm{M} / \mathrm{F})\end{array}$ & $\begin{array}{l}\text { Mean } \\
\text { age }\end{array}$ & $\begin{array}{l}\text { Matching } \\
\text { items }\end{array}$ & $\begin{array}{l}\text { Median } \\
\text { follow-up time } \\
\text { (range), mo. }\end{array}$ & $\begin{array}{l}\text { Detailed data of } \\
\text { staging }\end{array}$ & $\begin{array}{l}\text { Histologic } \\
\text { type } \\
(\mathrm{WHO})\end{array}$ & $\begin{array}{l}\text { High } \\
\text { quality }\end{array}$ & $\begin{array}{l}\text { Level of } \\
\text { evidence }\end{array}$ \\
\hline Guo/2016 [14] & $\begin{array}{l}\text { Mainland, } \\
\text { China }\end{array}$ & English & $2005-2010$ & $\mathrm{R}$ & $311(220 / 91)$ & NR & NR & $57.0(5.0-105.0)$ & AJCC-2010 II & I-III & No & 4 \\
\hline $\begin{array}{l}\text { Zhang/2015 } \\
{[16]}\end{array}$ & $\begin{array}{l}\text { Mainland, } \\
\text { China }\end{array}$ & English & 2009-2012 & $\mathrm{R}$ & $305^{\mathrm{a}}(222 / 83)$ & NR & $\begin{array}{l}1,2,3,4,5 \\
6,7,8\end{array}$ & $37.3(8.0-58.8)$ & $\begin{array}{l}\text { AJCC-2010 } \\
\text { II+T3N0M0 (21.4\%) }\end{array}$ & I-III & Yes & $2 b$ \\
\hline $\mathrm{Xu} / 2015$ [15] & $\begin{array}{l}\text { Mainland, } \\
\text { China }\end{array}$ & English & 2009-2011 & $\mathrm{R}$ & $86(63 / 23)$ & 50.5 & $\begin{array}{l}1,2,3,4,7 \\
9\end{array}$ & $37.4(4.8-66.2)$ & AJCC-2002 II & NR & Yes & $2 b$ \\
\hline Su/2015 [17] & $\begin{array}{l}\text { Mainland, } \\
\text { China }\end{array}$ & English & 2005-2010 & $\mathrm{R}$ & $249(178 / 71)$ & NR & $\begin{array}{l}1,2,3,4,10, \\
11\end{array}$ & $59.4(4.0-115.7)$ & AJCC-2010 II & II-III & Yes & 4 \\
\hline $\begin{array}{l}\text { Kang/2015 } \\
{[12]}\end{array}$ & $\begin{array}{l}\text { South } \\
\text { Korea }\end{array}$ & English & 2004-2011 & $\mathrm{R}$ & $138(98 / 40)$ & NR & $1,2,3,4$ & $48.0(7.0-79.0)$ & AJCC-2002 II & I-III & No & 4 \\
\hline Luo/2014 [13] & $\begin{array}{l}\text { Mainland, } \\
\text { China }\end{array}$ & English & 2006-2010 & $\mathrm{R}$ & $69(38 / 31)$ & 42.0 & NR & $34.0(12.0-64.0)$ & $\begin{array}{l}\text { AJCC-2002 } \\
\text { II+T1N0M0 (24.6\%) }\end{array}$ & II-III & No & 4 \\
\hline $\mathrm{Xu} / 2011$ [6] & $\begin{array}{l}\text { Mainland, } \\
\text { China }\end{array}$ & English & 2000-2003 & $\mathrm{R}$ & $392(293 / 99)$ & 48.0 & $\begin{array}{l}1,2,3,4,7 \\
12\end{array}$ & $66.0(2.4-117.1)$ & AJCC-2002 II & I-III & Yes & $2 b$ \\
\hline Song/2008 [5] & $\begin{array}{l}\text { South } \\
\text { Korea }\end{array}$ & English & 1986-2004 & $\mathrm{R}$ & $43^{b}(30 / 13)$ & 50.0 & $\begin{array}{l}1,2,3,4,5 \\
6\end{array}$ & $\begin{array}{l}124.5 \\
(5.0-239.0)\end{array}$ & AJCC-1997 IIb & I-III & No & 4 \\
\hline $\begin{array}{l}\text { Chua/2006 } \\
{[28]}\end{array}$ & $\begin{array}{l}\text { Hong } \\
\text { Kong, } \\
\text { China }\end{array}$ & English & 1989-1994 & $\mathrm{R}$ & $208(145 / 63)$ & 43.6 & $1,2,4,5,6$ & 67.0 (NR) & AJCC-1997 IIb & I-III & Yes & $2 b$ \\
\hline Chen/2011 [4] & $\begin{array}{l}\text { Mainland, } \\
\text { China }\end{array}$ & English & 2003-2007 & RCT & $230(166 / 64)$ & 42.5 & $\begin{array}{l}1,2,3,4,5 \\
6,9\end{array}$ & $60.0(5.0-87.0)$ & Chinese-1992 II & II-III & Yes & $2 b$ \\
\hline $\begin{array}{l}\text { Chen/2015 } \\
\text { [27] }\end{array}$ & $\begin{array}{l}\text { Mainland, } \\
\text { China }\end{array}$ & Chinese & 2007-2014 & $\mathrm{R}$ & 107 (NR) & NR & $\begin{array}{l}1,2,3,4,5 \\
6,7\end{array}$ & $47.0(6.0-89.0)$ & AJCC-2010 II & III & Yes & $2 b$ \\
\hline
\end{tabular}

Abbreviations: AJCC: American Joint Committee on Cancer; WHO: World Health Organization; R: retrospective; RCT: randomized controlled trial; M: male; F: female; mo.: months; NR: not reported.

a This study enrolled 440 patients, but only 305 patients were analyzed using propensity score matching.

b This study enrolled 60 patients with AJCC-1997 stage I-II NPC; we extracted the available data of 43 patients with stage IIb NPC.

${ }^{c}$ Matching items: 1 = age; 2 = sex; $3=$ pathology; $4=$ T stage; $5=\mathrm{N}$ stage; $6=$ stage; $7=$ Karnofsky score; $8=$ pre-treatment plasma Epstein-Barr Virus DNA; $9=$ retropharyngeal lymph node involvement; 10 = cigarette smoking; $11=$ alcohol consumption; 12 = lactate dehydrogenase level.

Table 2. Summary of radiotherapy parameters and chemotherapy regimens of included studies

\begin{tabular}{|c|c|c|c|c|c|}
\hline \multirow{2}{*}{$\begin{array}{l}\text { First author } \\
\text { / year }\end{array}$} & \multicolumn{2}{|c|}{ No. of patients } & \multirow[t]{2}{*}{ Radiotherapy } & \multicolumn{2}{|l|}{ Chemotherapy } \\
\hline & RT & CRT & & Concurrent chemotherapy & Induction and/or adjuvant chemotherapy \\
\hline $\begin{array}{l}\text { Guo/2016 } \\
{[14]}\end{array}$ & 66 & 245 & IMRT: 69.75 Gy (2.25 Gy/fx/d, 5 fx/wk) & $(1-6)^{*} \mathrm{DDP} 80 \mathrm{mg} / \mathrm{m}^{2}(\mathrm{~d} 1-3) ; \mathrm{DDP}$ and PTX & $\begin{array}{l}\text { IC and/or AC (55.8\%): }(1-6)^{*} \text { DDP } 80 \\
\mathrm{mg} / \mathrm{m}^{2}(\mathrm{~d} 1-3) \text { and PTX } 135 \mathrm{mg} / \mathrm{m} 2(\mathrm{~d} 1) \\
\text { GEM } 1000 \mathrm{mg} / \mathrm{m}^{2}(\mathrm{~d} 1, \mathrm{~d} 8)\end{array}$ \\
\hline $\begin{array}{l}\text { Zhang/2015 } \\
\text { [16] }\end{array}$ & 112 & 193 & $\begin{array}{l}\text { IMRT: } 66-72 \text { Gy }(2.27 \mathrm{~Gy} / \mathrm{fx} / \mathrm{d}, 5 \mathrm{fx} / \mathrm{wk}) \text {; } \\
\text { additional intracavitary irradiation for } \\
\text { local tumor persistence }\end{array}$ & $\begin{array}{l}(\geq 2)^{*} \text { DDP or Nedaplatin } 80-100 \mathrm{mg} / \mathrm{m}^{2} \\
\text { 3-weekly; }(\geq 5)^{*} \text { DDP } 30-50 \mathrm{mg} / \mathrm{m}^{2} \text { or } \\
\text { Nedaplatin } 20-30 \mathrm{mg} / \mathrm{m}^{2} \text { or CBP } 100 \\
\mathrm{mg} / \mathrm{m}^{2} ;(3-6)^{*} \text { DOC } 15-35 \mathrm{mg} / \mathrm{m}^{2} \text {, weekly }\end{array}$ & IC and $\mathrm{AC}(28 \%)$ : NR \\
\hline $\begin{array}{l}\mathrm{Xu} / 2015 \\
{[15]}\end{array}$ & 43 & 43 & $\begin{array}{l}\text { IMRT: } 66 \mathrm{~Gy}(2.2 \mathrm{~Gy} / \mathrm{fx}, 5 \mathrm{fx} / \mathrm{wk}) \text {; local } \\
\text { dose boost for residual primary lesion or } \\
\text { neck LN+ }\end{array}$ & $(5-6)^{*} \mathrm{DDP} 40 \mathrm{mg} / \mathrm{m}^{2}$, weekly & None \\
\hline $\begin{array}{l}\mathrm{Su} / 2015 \\
{[17]}\end{array}$ & 106 & 143 & $\begin{array}{l}\text { IMRT: } 68 \text { Gy }(2.26 \mathrm{~Gy} / \mathrm{fx} / \mathrm{d}, 5 \mathrm{fx} / \mathrm{wk}) ; 50 \\
\text { Gy }(2 \mathrm{~Gy} / \mathrm{fx} / \mathrm{d}) \text { for lower neck and } \\
\text { supraclavicular fossae }\end{array}$ & $\begin{array}{l}(2-6)^{*} \text { Platinum single-agent, } 3 \text {-weekly or } \\
\text { weekly; PTX or PF or TP regime }\end{array}$ & None \\
\hline $\begin{array}{l}\text { Kang/2015 } \\
{[12]}\end{array}$ & 41 & 97 & $\begin{array}{l}\text { IMRT+2D-RT (2\%): 64-74.2 Gy }(2.12 \\
\text { Gy/fx/d, } 5 \mathrm{fx} / \mathrm{wk})\end{array}$ & $\begin{array}{l}\text { DDP weekly or 3-weekly or 4-weekly; PF } \\
\text { regime }\end{array}$ & IC (17\%): DDP+5-FU or DOC or both \\
\hline $\begin{array}{l}\text { Luo/2014 } \\
{[13]}\end{array}$ & 25 & 44 & $\begin{array}{l}\text { IMRT: } 68-72 \text { Gy }(2.2 \mathrm{~Gy} / \mathrm{fx} / \mathrm{d}, 5 \mathrm{fx} / \mathrm{wk}) \text {; } \\
\text { 66-70 Gy for neck LN+ }\end{array}$ & DDP $80-100$ mg/m² (d1-3), 3-weekly & None \\
\hline $\mathrm{Xu} / 2011[6]$ & 211 & 181 & $\begin{array}{l}\text { 2D-RT: } 70 \text { Gy }(2.0 \mathrm{~Gy} / \mathrm{fx} / \text { day, } 5 \mathrm{fx} / \mathrm{wk}) \text {; } \\
66-70 \text { Gy for neck LN+ }\end{array}$ & $\begin{array}{l}3^{*} \mathrm{DDP} 100 \mathrm{mg} / \mathrm{m}^{2}(\mathrm{~d} 1, \mathrm{~d} 22, \mathrm{~d} 43) ; 2 * \mathrm{DDP} \\
100 \mathrm{mg} / \mathrm{m}^{2}(\mathrm{~d} 1, \mathrm{~d} 22)\end{array}$ & None \\
\hline $\begin{array}{l}\text { Song/2008 } \\
\text { [5] }\end{array}$ & 22 & 21 & $\begin{array}{l}\text { 2D-RT: 66.6-72 Gy (1.8-2.0 Gy/fx/day, } 5 \\
\mathrm{fx} / \text { wk); } 54-72 \text { Gy for neck LN+ }\end{array}$ & None & $\begin{array}{l}\text { IC: } 3^{*} \text { DDP } 100 \mathrm{mg} / \mathrm{m}^{2}(\mathrm{~d} 1) ; 3^{*} 5-\mathrm{FU} 1000 \\
\mathrm{mg} / \mathrm{m}^{2}(\mathrm{~d} 1-5)\end{array}$ \\
\hline $\begin{array}{l}\text { Chua/2006 } \\
{[28]}\end{array}$ & 110 & 98 & $\begin{array}{l}\text { 2D-RT: 66-74 Gy }(2.0 \mathrm{~Gy} / \mathrm{fx} / \text { day, } 5 \\
\mathrm{fx} / \mathrm{wk}) ; 60-76 \text { Gy for neck LN+ }\end{array}$ & None & $\begin{array}{l}\text { IC: }(2-3)^{*} \text { DDP } 60 \mathrm{mg} / \mathrm{m}^{2}(\mathrm{~d} 1) \text { and EPI } 110 \\
\mathrm{mg} / \mathrm{m}^{2}(\mathrm{~d} 1) ; \mathrm{DDP} 100 \mathrm{mg} / \mathrm{m}^{2}(\mathrm{~d} 1) \text { and } \\
\text { 5-FU } 800 \mathrm{mg} / \mathrm{m}^{2}(\mathrm{~d} 1-5) \text { and BLM } 10 \\
\mathrm{mg} / \mathrm{m}^{2}(\mathrm{~d} 1, \mathrm{~d} 5), 3 \text {-weekly }\end{array}$ \\
\hline $\begin{array}{l}\text { Chen/2011 } \\
{[4]}\end{array}$ & 114 & 116 & $\begin{array}{l}\text { 2D-RT: } 68-70 \text { Gy }(2.0 \mathrm{~Gy} / \mathrm{fx} / \text { day, } 5 \\
\mathrm{fx} / \mathrm{wk}) ; 60-62 \mathrm{~Gy} \text { for neck LN+ }\end{array}$ & $(6-8)^{*}$ DDP $30 \mathrm{mg} / \mathrm{m}^{2}(\mathrm{~d} 1)$, weekly & None \\
\hline $\begin{array}{l}\text { Chen/2015 } \\
\text { [27] }\end{array}$ & 51 & 56 & $\begin{array}{l}\text { IMRT+2D-RT ( } 45.8 \%): 66-70 \text { Gy }(2.0 \\
\text { Gy/fx/day, } 5 \text { fx/wk); } 60-70 \text { Gy for neck } \\
\text { LN+ }\end{array}$ & $\begin{array}{l}(2-3)^{*} \mathrm{DDP} 80 \mathrm{mg} / \mathrm{m}^{2} \text { or } 100 \mathrm{mg} / \mathrm{m}^{2}(\mathrm{~d} 1-3) \text {, } \\
\text { 3-weekly }\end{array}$ & None \\
\hline
\end{tabular}




\section{Assessment of the included studies}

The two investigators showed a good agreement in their assessment of the study quality of ten retrospective studies, with a kappa coefficient of 0.912 $(P<0.001)$ (Supplementary Table S1). The general quality of the ten retrospective studies was fair. Six of the ten studies had scores $\geq 6[6,15-17,27,28]$. Five studies reached evidence level $2 b$, of which, one study used the propensity score matching method with two well-matched cohorts to mimic a randomized trial [16], and one study implemented the 1-to-1 paired comparison method [15]. The remaining five studies (level of evidence: 4) consisted of four typically-designed retrospective studies [5, 13, 14, 17], and one study with a complicated design compromising its reliability [12]. As for the only one RCT, the study quality was good (Supplementary Table S2) [4]. This study complied with the intention-to-treat principle, avoided selective outcome reporting and assessed each outcome adequately. All patients were randomly assigned to two groups with allocation concealment. However, it was unclear whether the blind method was used.

\section{CRT versus $R T$ alone}

The overall meta-analysis of primary outcomes is summarized in Table 3. Pooling the data assessing OS for 2138 patients from all included studies revealed marginally significant difference between CRT and RT alone $(\mathrm{HR}=0.67,95 \% \mathrm{CI}=0.45-0.98, P=$ $0.04)$ and significant between-study heterogeneity $(P$ $=0.09$ ). Ten studies assessed LRRFS in 2069 patients, and one study reported local relapse-free survival as outcome [13]. The pooled data revealed a significantly improved LRRFS for CRT compared to RT alone (HR $=0.61,95 \% \mathrm{CI}=0.46-0.80, P=0.0003)$. Between-study heterogeneity was non-significant $(P=0.10)$. In pooled analysis of 2138 patients across the included studies, DMFS was not statistically different between CRT and RT alone (HR $=0.83,95 \% \mathrm{CI}=0.52-1.31, P=$ $0.41)$, while the between-study heterogeneity was significant $(P=0.01)$.

Secondary outcomes could be extracted from three to five studies, representing 640 to 1262 cases with grade 3-4 acute toxicities (Table 3) [4, 6, 15-17]. The pooled outcomes indicated that CRT has higher frequencies of leukopenia $(\mathrm{RR}=6.40,95 \% \mathrm{CI}=$ 1.77-23.15, $P=0.005)$, mucositis $(\mathrm{RR}=1.41,95 \% \mathrm{CI}=$ 1.03-1.93, $P=0.03)$ and nausea $(\mathrm{RR}=10.61,95 \% \mathrm{CI}=$ 3.49-32.27, $P<0.0001$ ) than RT alone.

\section{CRT versus IMRT alone and CRT versus 2D-RT alone}

Two RT subgroups, 2D-RT alone and IMRT alone, were generated to compare with CRT in subgroup analyses. Seven retrospective studies enrolling 1265 patients compared CRT with IMRT alone $[12-17,27]$. Unlike the overall meta-analysis, CRT did not obtain improved OS ( $\mathrm{HR}=0.69,95 \% \mathrm{CI}=$ $0.41-1.13, P=0.14$; Figure 2A), LRRFS (HR $=0.49,95 \%$ $\mathrm{CI}=0.24-1.02, P=0.06$; Figure $3 \mathrm{~A})$ or DMFS $(\mathrm{HR}=$ $1.03,95 \% \mathrm{CI}=0.66-1.61, P=0.89$; Figure $4 \mathrm{~A}$ ) compared to IMRT alone. Moreover, non-significant between-study heterogeneity was observed in OS $(P=$ $\left.0.18, I^{2}=32 \%\right)$ and DMFS $\left(P=0.21, I^{2}=29 \%\right)$; moderate between-study heterogeneity was reported in LRRFS $\left(P=0.07, I^{2}=52 \%\right)$. Three of the seven studies also investigated secondary outcomes among 640 patients [15-17]. The result showed that CRT could obtain higher frequencies of grade 3-4 leukopenia $(P<$ $0.0001)$ and thrombocytopenia $(P=0.02)$ than IMRT alone.

Table 3. Results of the meta-analysis for the comparison of CRT and RT alone

\begin{tabular}{|c|c|c|c|c|c|c|c|c|c|c|}
\hline \multirow[t]{2}{*}{ Outcome } & \multirow{2}{*}{$\begin{array}{l}\text { No. of } \\
\text { studies }\end{array}$} & \multirow{2}{*}{$\begin{array}{l}\text { No. of } \\
\text { RT pts }\end{array}$} & \multirow{2}{*}{$\begin{array}{l}\text { No. of } \\
\text { CRT pts }\end{array}$} & \multirow{2}{*}{$\begin{array}{l}\text { No. of all } \\
\text { pts }\end{array}$} & \multirow{2}{*}{$\begin{array}{l}\mathrm{HR} / \mathrm{RR} \\
(95 \% \mathrm{CI})\end{array}$} & \multirow[t]{2}{*}{$P$-value ${ }^{\mathrm{b}}$} & \multicolumn{4}{|c|}{ Study heterogeneity } \\
\hline & & & & & & & $\overline{x^{2}}$ & $d f$ & $I^{2}(\%)$ & $P$-value \\
\hline \multicolumn{11}{|l|}{ Primary outcomes } \\
\hline Overall survival & 11 & 901 & 1237 & 2138 & $0.67[0.45,0.98]^{\mathrm{a}}$ & 0.04 & 16.34 & 10 & 39 & 0.09 \\
\hline Loco-regionally relapse-free survival & 10 & 876 & 1193 & 2069 & $0.61[0.46,0.80]^{\mathrm{a}}$ & 0.0003 & 14.81 & 9 & 39 & 0.10 \\
\hline Distant metastasis-free survival & 11 & 901 & 1237 & 2138 & $0.83[0.52,1.31]^{\mathrm{a}}$ & 0.41 & 23.14 & 10 & 57 & 0.01 \\
\hline \multicolumn{11}{|l|}{ Secondary outcomes } \\
\hline Leukopenia & 5 & 586 & 676 & 1262 & $6.40[1.77,23.15]$ & 0.005 & 10.17 & 4 & 61 & 0.04 \\
\hline Anemia & 3 & 261 & 379 & 640 & $0.96[0.16,5.79]$ & 0.97 & 0.06 & 2 & 0 & 0.81 \\
\hline Thrombocytopenia & 5 & 586 & 676 & 1262 & $1.67[0.91,3.06]$ & 0.10 & 5.27 & 4 & 24 & 0.26 \\
\hline Liver dysfunction & 4 & 375 & 495 & 870 & $1.74[0.87,3.48]$ & 0.12 & 1.93 & 3 & 0 & 0.38 \\
\hline Renal impairment & 4 & 375 & 495 & 870 & $4.51[0.79,25.66]$ & 0.09 & 0.09 & 3 & 0 & 0.77 \\
\hline Mucositis & 5 & 586 & 676 & 1262 & $1.41[1.03,1.93]$ & 0.03 & 11.34 & 4 & 65 & 0.02 \\
\hline Dermatitis & 5 & 586 & 676 & 1262 & $1.37[0.84,2.23]$ & 0.21 & 1.87 & 4 & 0 & 0.60 \\
\hline Nausea & 4 & 543 & 633 & 1176 & $10.61[3.49,32.27]$ & $<0.0001$ & 0.88 & 3 & 0 & 0.83 \\
\hline
\end{tabular}

Abbreviations: CRT: chemoradiotherapy; RT: radiotherapy; pts: patients; HR: hazard ratio; RR: risk ratio; CI: confidence interval; $d f$ : degrees of freedom.

a Hazard ratio.

bStatistically significant results are shown in bold. 


\section{A}

\begin{tabular}{lccrc} 
Study or Subgroup & log[Hazard Ratio] & SE & Weight & IV, Fixed, 95\% C \\
\hline CRT vs. IMRT alone & & & & \\
Chen-2015 & -1.4 & 0.86 & $8.8 \%$ & $0.25[0.05,1.33]$ \\
Guo-2016 & -0.12 & 0.48 & $28.4 \%$ & $0.89[0.35,2.27]$ \\
Kang-2015 & -0.69 & 0.61 & $17.6 \%$ & $0.50[0.15,1.66]$ \\
Luo-2014 & -2.14 & 1.04 & $6.0 \%$ & $0.12[0.02,0.90]$ \\
Su-2015 & 0.53 & 0.49 & $27.2 \%$ & $1.70[0.65,4.44]$ \\
Xu-2015 & -0.94 & 1.23 & $4.3 \%$ & $0.39[0.04,4.35]$ \\
Zhang-2015 & -0.97 & 0.93 & $7.6 \%$ & $0.38[0.06,2.35]$ \\
& & & & \\
Total (95\% CI) & & & $100.0 \%$ & $0.69[0.41,1.13]$ \\
Heterogeneity: Chi ${ }^{2}=8.88, d f=6(P=0.18): 1^{2}=32 \%$ & &
\end{tabular}

Heterogeneity: $\mathrm{Chi}^{2}=8.88, \mathrm{df}=6(\mathrm{P}=0.18) ; \mathrm{I}^{2}=32 \%$

Test for overall effect: $Z=1.48(P=0.14)$

Hazard Ratio Hazard Ratio

IV, Fixed, $95 \% \mathrm{Cl}$

\section{B}

\begin{tabular}{|c|c|c|c|c|}
\hline Study or Subgroup & $\log [$ Hazard Ratio] & SE & Weight & IV, Random, $95 \%$ \\
\hline \multicolumn{5}{|l|}{ CRT vs. 2D-RT alone } \\
\hline Chen-2011 & -1.2 & 0.45 & $19.6 \%$ & $0.30[0.12,0.73]$ \\
\hline Chua-2006 & -0.53 & 0.27 & $30.5 \%$ & $0.59[0.35,1.00]$ \\
\hline Song-2008 & 0.36 & 0.54 & $15.7 \%$ & $1.43[0.50,4.13]$ \\
\hline Xu-2011 & -0.06 & 0.22 & $34.1 \%$ & $0.94[0.61,1.45]$ \\
\hline Total $(95 \% \mathrm{CI})$ & & & $100.0 \%$ & $0.70[0.41,1.18]$ \\
\hline
\end{tabular}

Hazard Ratio Hazard Ratio

IV, Random, $95 \% \mathrm{CI}$

$\mathrm{Cl}$
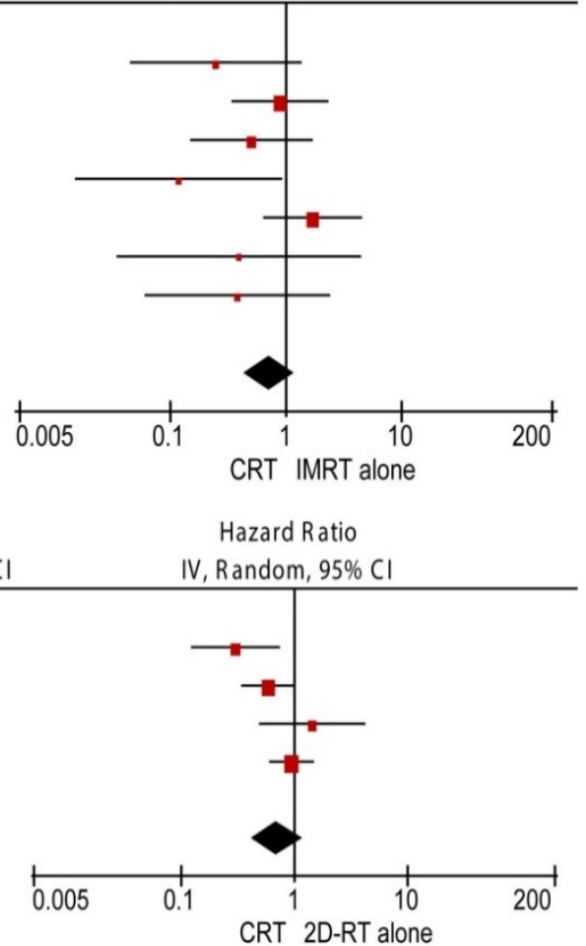

Figure 2. Forest plot and meta-analysis of overall survival (OS) in subgroup analysis. (A) CRT versus IMRT alone; (B) CRT versus 2D-RT alone. Squares are the point estimates of the HRs with the $95 \% \mathrm{Cls}$ indicated by horizontal bars. Diamonds are the summary estimates and $95 \%$ Cls from the pooled studies. CRT: chemoradiotherapy; IMRT: intensity modulated radiotherapy; 2D-RT: two-dimensional radiotherapy; Cl: confidence interval; SE: standard error; IV: inverse variance method.

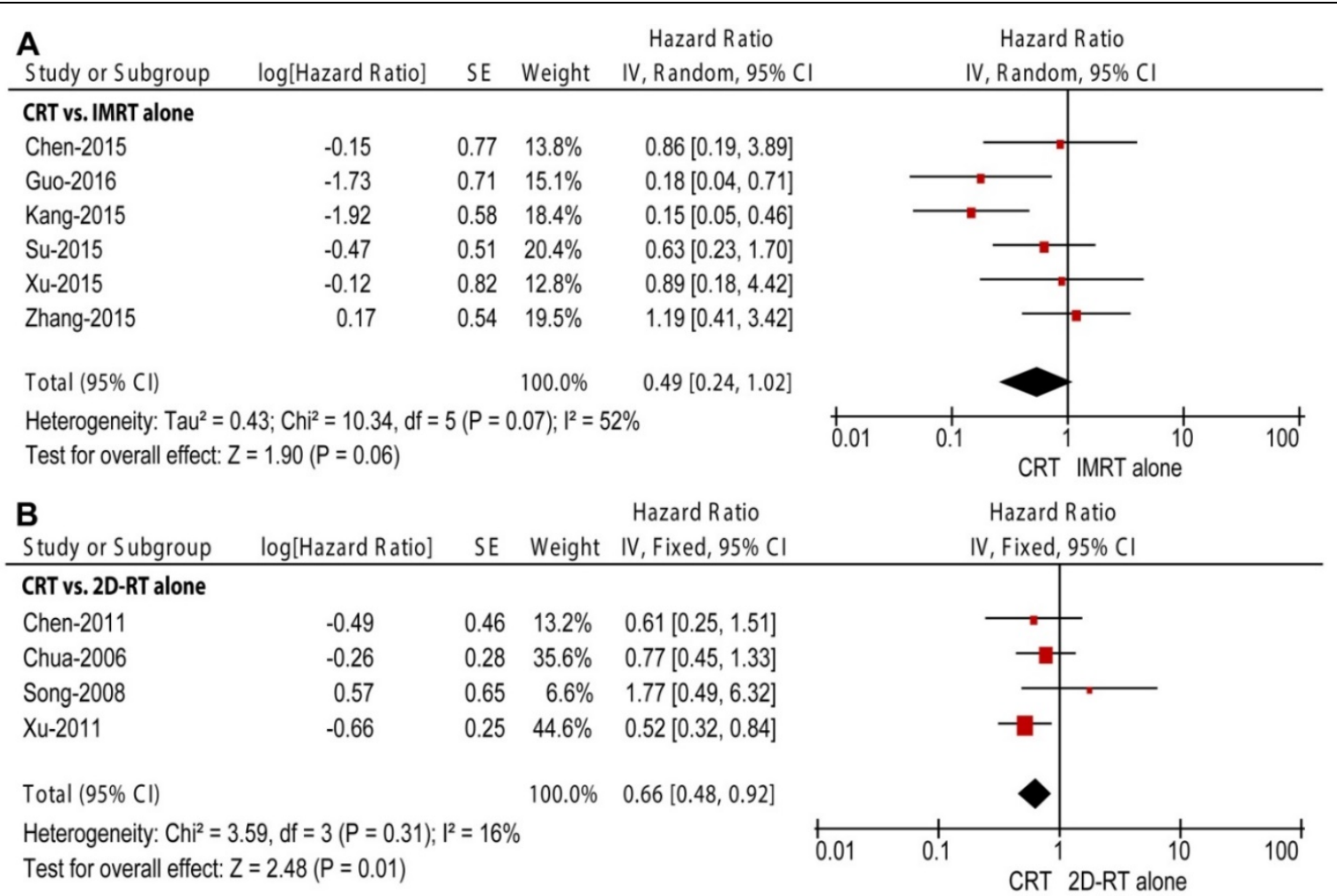

Figure 3. Forest plot and meta-analysis of loco-regional relapse-free survival (LRRFS) in subgroup analysis. (A) CRT versus IMRT alone; (B) CRT versus 2D-RT alone. Squares are the point estimates of the HRs with the $95 \% \mathrm{Cls}$ indicated by horizontal bars. Diamonds are the summary estimates and $95 \% \mathrm{Cls}$ from the pooled studies. CRT: chemoradiotherapy; IMRT: intensity modulated radiotherapy; 2D-RT: two-dimensional radiotherapy; Cl: confidence interval; SE: standard error; IV: inverse variance method. 
Four studies enrolling 873 patients were included in the subgroup of CRT versus 2D-RT alone, including three retrospective studies and one RCT $[4-6,28]$. The pooled data revealed non-significant differences in OS and DMFS (HR $=0.70,95 \% \mathrm{CI}=$ $0.41-1.18, P=0.18$, Figure $2 \mathrm{~B} ; \mathrm{HR}=0.69,95 \% \mathrm{CI}=$ $0.30-1.59, P=0.39$, Figure $4 \mathrm{~B}$, respectively) between the two modalities. The pooled OS and DMFS were accompanied with moderate and high between-study heterogeneity $\left(P=0.06, I^{2}=59 \% ; P=0.003, I^{2}=78 \%\right.$, respectively). Moreover, CRT showed significantly higher LRRFS than 2D-RT alone $(\mathrm{HR}=0.66,95 \% \mathrm{CI}=$ $0.48-0.92, \quad P=0.01$, Figure $3 \mathrm{~B})$; no significant between-study heterogeneity was observed $(P=0.31$, $\left.I^{2}=16 \%\right)$.

\section{Sensitivity analysis and assessment of publication bias}

Sensitivity analysis was carried out to assess stability of the study results of CRT versus IMRT alone (Table 4). The sensitivity analysis that individually excluded one study published in Chinese
[27], two studies enrolling less than one hundred patients [13, 15], and three studies with relatively low-quality [12-14], yielded no changes in the significance of any survival outcome as the original subgroup analysis of CRT versus IMRT alone. Meanwhile, $I^{2}$-values of all pooled outcomes in the sensitivity analysis of high-quality studies were $0 \%$. Considering that different CRT schedules were used in comparison to IMRT alone, we individually included four studies using CCRT $[13,15,17,27]$ and three studies using CCRT plus IC and/or AC [12, 14, 16] in sensitivity analysis. All pooled survival outcomes still remained non-significant. Thus, the results of CRT versus IMRT alone were of high stability.

As for the overall meta-analysis, no significant publication bias was observed in OS, LRRFS or DMFS $(P=0.186,0.967,0.774$, respectively). A funnel plot was created for OS; all studies were located inside the 95\% CIs with a symmetric distribution (Figure 5).

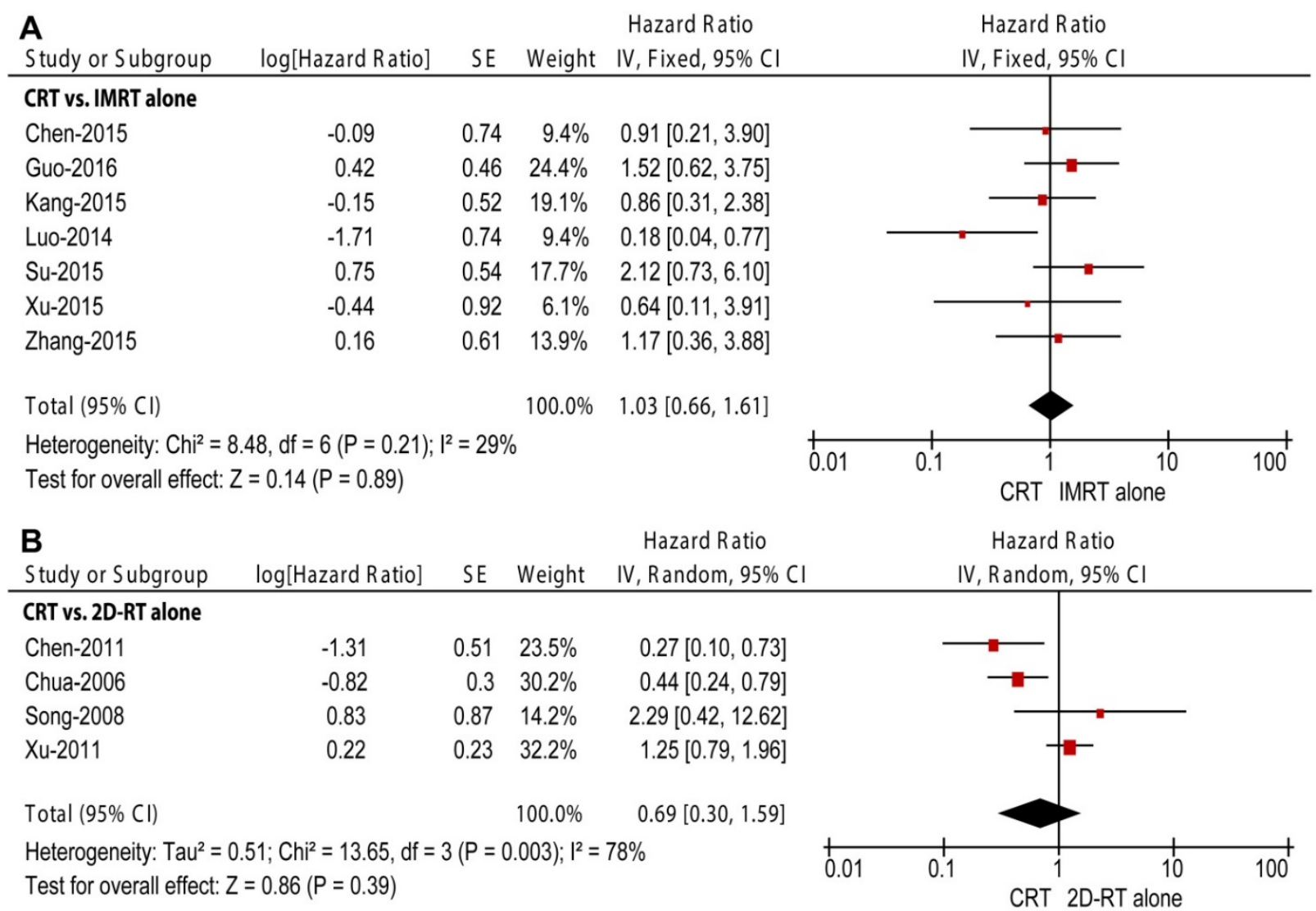

Figure 4. Forest plot and meta-analysis of distant metastasis-free survival (DMFS) in subgroup analysis. (A) CRT versus IMRT alone; (B) CRT versus 2D-RT alone. Squares are the point estimates of the HRs with the $95 \% \mathrm{Cls}$ indicated by horizontal bars. Diamonds are the summary estimates and $95 \%$ Cls from the pooled studies. CRT: chemoradiotherapy; IMRT: intensity modulated radiotherapy; 2D-RT: two-dimensional radiotherapy; Cl: confidence interval; SE: standard error; IV: inverse variance method. 
Table 4. Sensitivity analysis for the comparison of CRT and IMRT alone.

\begin{tabular}{|c|c|c|c|c|c|c|c|c|c|c|}
\hline \multirow[t]{2}{*}{ Outcome } & \multirow{2}{*}{$\begin{array}{l}\text { No. of } \\
\text { studies }\end{array}$} & \multirow{2}{*}{$\begin{array}{l}\text { No. of } \\
\text { IMRT pts }\end{array}$} & \multirow{2}{*}{$\begin{array}{l}\text { No. of } \\
\text { CRT pts }\end{array}$} & \multirow{2}{*}{$\begin{array}{l}\text { No. of all } \\
\text { pts }\end{array}$} & \multirow{2}{*}{$\begin{array}{l}\text { HR } \\
(95 \% \mathrm{CI})\end{array}$} & \multirow[t]{2}{*}{$P$-value } & \multicolumn{4}{|c|}{ Study heterogeneity } \\
\hline & & & & & & & $\overline{x^{2}}$ & $d f$ & $I^{2}(\%)$ & $P$-value \\
\hline \multicolumn{11}{|l|}{ English publications } \\
\hline Overall survival & 6 & 393 & 765 & 1158 & $0.76[0.45,1.28]$ & 0.30 & 7.33 & 5 & 32 & 0.20 \\
\hline Loco-regionally relapse-free survival & 5 & 368 & 721 & 1089 & $0.45[0.19,1.04]$ & 0.06 & 9.76 & 4 & 59 & 0.04 \\
\hline $\begin{array}{l}\text { Distant metastasis-free survival } \\
\text { Sample size }>100 \text { patients }\end{array}$ & 6 & 393 & 765 & 1158 & $1.05[0.66,1.67]$ & 0.85 & 8.45 & 5 & 41 & 0.13 \\
\hline Overall survival & 5 & 376 & 734 & 1110 & $0.79[0.47,1.35]$ & 0.39 & 5.51 & 4 & 27 & 0.24 \\
\hline Loco-regionally relapse-free survival & 5 & 376 & 734 & 1110 & $0.45[0.19,1.03]$ & 0.06 & 9.78 & 4 & 59 & 0.04 \\
\hline $\begin{array}{l}\text { Distant metastasis-free survival } \\
\text { High-quality studies }\end{array}$ & 5 & 376 & 734 & 1110 & $1.30[0.80 .2 .11]$ & 0.29 & 1.82 & 4 & 0 & 0.77 \\
\hline Overall survival & 4 & 312 & 435 & 747 & $0.63[0.22,1.81]$ & 0.39 & 5.21 & 3 & 42 & 0.16 \\
\hline Loco-regionally relapse-free survival & 4 & 312 & 435 & 747 & $0.85[0.47,1.57]$ & 0.61 & 0.75 & 3 & 0 & 0.86 \\
\hline Distant metastasis-free survival & 4 & 312 & 435 & 747 & $1.29[0.67,2.47]$ & 0.44 & 1.65 & 3 & 0 & 0.65 \\
\hline \multicolumn{11}{|l|}{ Studies using CCRT } \\
\hline Overall survival & 4 & 225 & 286 & 511 & $0.45[0.11,1.75]$ & 0.25 & 7.90 & 3 & 62 & 0.05 \\
\hline Loco-regionally relapse-free survival & 3 & 200 & 242 & 442 & $0.73[0.35,1.52]$ & 0.40 & 0.19 & 2 & 0 & 0.91 \\
\hline Distant metastasis-free survival & 4 & 225 & 286 & 511 & $0.74[0.25,2.23]$ & 0.59 & 7.33 & 3 & 59 & 0.06 \\
\hline \multicolumn{11}{|l|}{ Studies using CCRT plus IC and/or AC } \\
\hline Overall survival & 3 & 219 & 535 & 754 & $0.65[0.33,1.29]$ & 0.22 & 0.94 & 2 & 0 & 0.63 \\
\hline Loco-regionally relapse-free survival & 3 & 219 & 535 & 754 & $0.32[0.08,1.31]$ & 0.11 & 8.26 & 2 & 76 & 0.02 \\
\hline Distant metastasis-free survival & 3 & 219 & 535 & 754 & $1.18[0.66,2.13]$ & 0.58 & 0.67 & 2 & 0 & 0.71 \\
\hline
\end{tabular}

Abbreviations: CRT: chemoradiotherapy; CCRT: concurrent chemoradiotherapy; IMRT: intensity modulated radiotherapy; IC: induction chemotherapy; AC: adjuvant chemotherapy; pts: patients; HR: hazard ratio; CI: confidence interval; $d f$ : degrees of freedom.

a Statistically significant results are shown in bold.

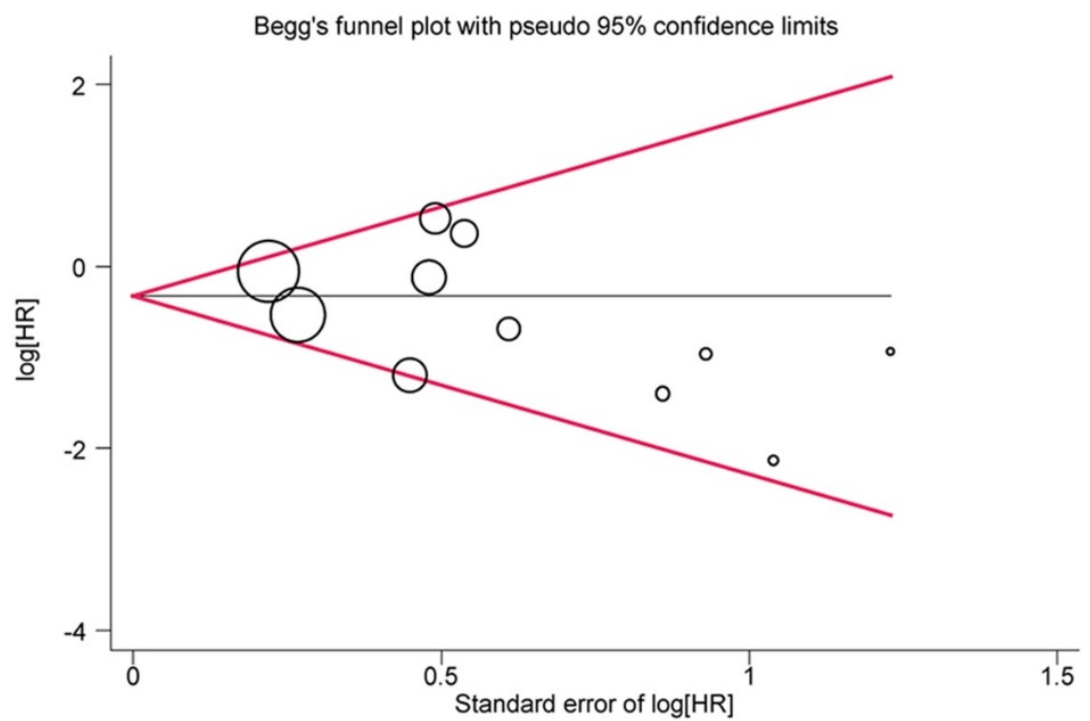

Figure 5. Begg's funnel plot for assessing publication bias of overall survival (OS) in overall meta-analysis. The size of the circles indicates the weight of each study. HR: hazard ratio.

\section{Discussion}

This meta-analysis enrolling 2138 patients with stage II NPC showed that CRT has significantly higher OS and LRRFS than RT alone. The improved LRRFS is due mainly to the superiority of CRT over 2D-RT alone. When it comes to the improved OS for CRT, drawing the conclusion should be discreet because of the marginal significance $(P=0.04)$ and obvious between-study heterogeneity. Besides, although both RT subgroups reported non-significant difference in OS compared to CRT, the result of IMRT alone was of high reliability, while the result of 2D-RT alone was accompanied with significant between-study heterogeneity. The inconsistence between studies comparing CRT and 2D-RT alone might be caused by different CRT schedules (CCRT, IC plus 2D-RT). Given the lack of number of studies and obvious heterogeneity, we did not perform further investigation (e.g., sensitivity analysis) for the subgroup of CRT versus 2D-RT alone. For the comparison of CRT and IMRT alone, the sensitivity analysis showed similar survival outcomes as the original analysis with excellent stability, which 
indicated the superiority of IMRT alone.

There are three possible explanations for the non-significant difference in survival outcomes between CRT and IMRT alone. Firstly, IMRT provides a higher local tumor control rate than conventional RT $[29,30]$; therefore, this advanced technique may narrow the potential therapeutic gains of CRT. The positive conclusion for CRT reached in the overall meta-analysis was associated with the relative efficacy of CRT compared to the sub-optimal treatment effects of 2D-RT alone [28]. Secondly, the increased frequencies of severe adverse reactions among patients treated with CRT may compromise the survival benefit of chemotherapy and result in more favorable outcomes for IMRT alone [31]. In the subgroup analysis of CRT versus IMRT alone, one study showed that IMRT alone could significantly improve OS [17]; this effect was associated with a higher incidence of grade 3-4 acute toxicities in the group of patients receiving CRT, especially non-hematological events such as liver dysfunction and renal impairment, compared to other included studies. Thirdly, it should be noted that patients with T2N1M0 NPC represent a special group at high risk of distant metastasis $[4,10,13]$. There is evidence that patients with T2N1M0 disease experience significantly poorer OS $(P=0.044)$ and DMFS $(P=$ 0.010) than those with T1N1M0 disease [14]. Moreover, some studies have indicated that it is difficult to eradicate micro-metastatic lesions using cisplatin-based regimens [32-34]. Therefore, assessment of patients with stage II NPC without precise population stratification may reduce the benefits of CRT to a non-significant effect.

Apart from the equivalent survival outcomes compared to CRT, IMRT also had fewer grade 3-4 hematological toxicities, which suggest better patient compliance and lower medical cost. Moreover, Tham et al. reported that IMRT alone had comparable survival outcomes with acceptable toxicities compared to CRT in stage IIb NPC [11]. Therefore, it seems that IMRT alone may be more suitable than CRT for patients with stage II NPC. Nonetheless, the benefit of CRT in LRRFS still calls for special attention and further discussion. Even though non-significant differences were observed for LRRFS between CRT and IMRT alone, the potential trend towards increased LRRFS for CRT and moderate between-study heterogeneity indicated a number of factors might contribute to and affect the results. Kang et al. reported that CRT could significantly improve LRRFS [12]; however, the OS, LRRFS and DMFS in this study were generally poor $(88.2 \%, 86.2 \%, 85.5 \%)$ compared to other included studies in which these rates generally exceed at least $90 \%$. Thus, objectively sub-optimal loco-regional control may be one possible factor that increased the apparent value of CRT. Moreover, the studies included in this meta-analysis adopted different regimens, recruited patients from different areas (endemic and non-endemic regions), and matched patients using different criteria. These variations could contribute to the between-study heterogeneity and discrepancies in survival outcomes. After the sequential exclusion of some studies in sensitivity analyses based on different matching criteria, IMRT alone was proved to be superior to CRT with excellent stability. Therefore, we can conclude on the basis of the stable outcomes that IMRT alone is better than CRT for patients with stage II NPC. Currently, several phase II-III trials are undertaking to confirm the efficacy of IMRT alone in stage II NPC compared to CRT (e.g., NCT02116231, NCT02610010), and the final results are awaiting to be reported.

The present meta-analysis has several limitations that must be taken into account. First, the main limitation is the inferior level of evidence. Only one study was RCT [4]; the remaining ten studies were retrospective observational articles with different designs, including a study by Kang et al. with a complicated design at the cost of reliability [12]. Second, the inclusion criteria for the meta-analysis need to be strictly amended to avoid inaccurate information. Two of the eleven studies individually included additional patients with T1N0M0 disease (24.6\%; AJCC-2002) [13] and T3N0M0 disease (21.4\%; AJCC-2010) [16]. As stage II NPC in the study including T1N0M0 disease overlapped with part of stage I NPC when patients were re-staged based on AJCC-2010, and the T3N0M0 subgroup was reported to have similar survival outcomes to patients with stage II $[35,36]$, we included the two studies in our meta-analysis. However, the inaccurate information could induce noticeable heterogeneity between studies and obscure conclusions of this meta-analysis. Third, several studies have suggested that the size of lymph node and pre-treatment Epstein-Barr virus (EBV)-deoxyribonucleic acid (DNA) levels are probably more important factors to show a difference with the addition of chemotherapy [37-39]. Thus, appropriate stratification of NPC patients should be established by incorporating lymph node classification, EBV-DNA and other biological/ molecular markers $[40,41]$. In the absence of detailed data of individuals, we cannot stratify patients with stage II NPC and failed to obtain the accurate conclusion. Further studies restricted to IMRT alone with individual patient data are awaited. Finally, only five studies directly reported survival data as HRs and the associated CIs [4, 5, 15, 16, 27]. Even though we calculated these values using the same methods 
designed by Tierney in all remaining studies, this may also result into bias and error [20].

\section{Conclusion}

The present meta-analysis showed that CRT is better than 2D-RT alone in stage II NPC with significant benefit in LRRFS. IMRT alone is superior to CRT with equivalent survival outcomes and fewer grade 3-4 acute toxicities. This meta-analysis is the first attempt to compare CRT with 2D-RT/IMRT alone in stage II NPC. In the future, well-designed RCTs comparing CRT and IMRT alone in stage II NPC and patient stratification based on lymph node classifications and pre-treatment EBV-DNA levels are needed.

\section{Supplementary Material}

Supplementary Figures.

http://www.jcancer.org/v08p0287s1.pdf

\section{Abbreviations}

NPC: nasopharyngeal carcinoma; RCT: randomized controlled trial; CRT: chemoradiotherapy; RT: radiotherapy; 2D-RT: two-dimensional radiotherapy; IMRT: intensity-modulated radiotherapy; CCRT: concurrent chemoradiotherapy; AC: adjuvant chemotherapy; IC: induction chemotherapy; PRISMA: Preferred Reporting Items for Systematic Reviews and Meta-analysis; MOOSE: Meta-analysis of Observational Studies in Epidemiology recommendations for study reporting; OS: overall survival; LRRFS: loco-regional relapse-free survival; DMFS: distant metastasis-free survival; AJCC: American Joint Committee on Cancer; WHO: World Health Organization; HR: hazard ratio; RR: risk ratio; $\mathrm{CI}$ : confidence interval; $\chi^{2}$ : Chi-square; $I^{2}$ : I-square; EBV: Epstein-Barr virus; DNA: deoxyribonucleic acid.

\section{Acknowledgements}

This work was supported by grants from the National Science \& Technology Pillar Program during the Twelfth Five-year Plan Period (No. 2014BAI09B10); the Health \& Medical Collaborative Innovation Project of Guangzhou City, China (No. 201400000001); the Planned Science and Technology Project of Guangdong Province (No. 2013B020400004); and the Science and Technology Project of Guangzhou City, China (No. 14570006).

\section{Competing Interests}

The authors have declared that no competing interest exists.

\section{References}

1. Wei WI, Sham JS. Nasopharyngeal carcinoma. Lancet. 2005; 365: 2041-54.

2. Pfister DG, Spencer S, Brizel DM, Burtness B, Busse PM, Caudell JJ, et al. Head and neck cancers, Version 2.2014. Clinical practice guidelines in oncology. J Natl Compr Canc Netw. 2014; 12: 1454-87.

3. Chan AT, Gregoire V, Lefebvre JL, Licitra L, Hui EP, Leung SF, et al. Nasopharyngeal cancer: EHNS-ESMO-ESTRO Clinical Practice Guidelines for diagnosis, treatment and follow-up. Ann Oncol. 2012; 23 (Suppl 7): S783-5.

4. Chen QY, Wen YF, Guo L, Liu H, Huang PY, Mo HY, et al. Concurrent chemoradiotherapy vs radiotherapy alone in stage II nasopharyngeal carcinoma: phase III randomized trial. J Natl Cancer Inst. 2011; 103: 1761-70.

5. Song $\mathrm{CH}$, Wu HG, Heo DS, Kim KH, Sung MW, Park CI. Treatment outcomes for radiotherapy alone are comparable with neoadjuvant chemotherapy followed by radiotherapy in early-stage nasopharyngeal carcinoma. Laryngoscope. 2008; 118: 663-70.

6. $\mathrm{Xu} \mathrm{T}, \mathrm{Hu} \mathrm{C}$, Wang $\mathrm{X}$, Shen $\mathrm{C}$. Role of chemoradiotherapy in intermediate prognosis nasopharyngeal carcinoma. Oral Oncol. 2011; 47: 408-13.

7. Blanchard P, Lee A, Marguet S, Leclercq J, Ng WT, Ma J, et al. Chemotherapy and radiotherapy in nasopharyngeal carcinoma: an update of the MAC-NPC meta-analysis. Lancet Oncol. 2015; 16: 645-55.

8. Zhang B, Mo Z, Du W, Wang Y, Liu L, Wei Y. Intensity-modulated radiation therapy versus 2D-RT or 3D-CRT for the treatment of nasopharyngeal carcinoma: A systematic review and meta-analysis. Oral Oncol. 2015; 51: 1041-6.

9. Wang TJC, Riaz N, Cheng SK, Lu JJ, Lee NY. Intensity-modulated radiation therapy for nasopharyngeal carcinoma: a review. J Radiat Oncol. 2012; 1: 129-46.

10. Su SF, Han F, Zhao C, Chen CY, Xiao WW, Li JX, et al. Long-term outcomes of early-stage nasopharyngeal carcinoma patients treated with intensity-modulated radiotherapy alone. Int J Radiat Oncol Biol Phys. 2012; 82: 327-33.

11. Tham IW, Lin S, Pan J, Han L, Lu JJ, Wee J. Intensity-modulated radiation therapy without concurrent chemotherapy for stage IIb nasopharyngeal cancer. Am J Clin Oncol. 2010; 33: 294-9.

12. Kang MK, Oh D, Cho KH, Moon SH, Wu H-G, Heo D-S, et al. Role of Chemotherapy in Stage II Nasopharyngeal Carcinoma Treated with Curative Radiotherapy. Cancer Res Treat. 2015; 47: 871-8.

13. Luo S, Zhao L, Wang J, Xu M, Li J, Zhou B, et al. Clinical outcomes for early-stage nasopharyngeal carcinoma with predominantly WHO II histology treated by intensity-modulated radiation therapy with or without chemotherapy in nonendemic region of China. Head Neck. 2014; 36: 841-7.

14. Guo Q, Lu T, Lin S, Zong J, Chen Z, Cui X, et al. Long-term survival of nasopharyngeal carcinoma patients with Stage II in intensity-modulated radiation therapy era. Jpn J Clin Oncol. 2016; 46: 241-7.

15. Xu T, Shen C, Zhu G, Hu C. Omission of Chemotherapy in Early Stage Nasopharyngeal Carcinoma Treated with IMRT: A Paired Cohort Study. Medicine. 2015; 94: e1457.

16. Zhang F, Zhang Y, Li WF, Liu X, Guo R, Sun Y, et al. Efficacy of Concurrent Chemotherapy for Intermediate Risk NPC in the Intensity-Modulated Radiotherapy Era: a Propensity-Matched Analysis. Sci Rep. 2015; 5: 173-8.

17. Su Z, Mao YP, Tang J, Lan XW, OuYang PY, Xie FY. Long-term outcomes of concurrent chemoradiotherapy versus radiotherapy alone in stage II nasopharyngeal carcinoma treated with IMRT: a retrospective study. Tumour Biol. 2015: 4429-38.

18. Stroup DF, Berlin JA, Morton SC, Olkin I, Williamson GD, Rennie D, et al. Meta-analysis of observational studies in epidemiology: a proposal for reporting. Meta-analysis Of Observational Studies in Epidemiology (MOOSE) group. JAMA. 2000; 283: 2008-12.

19. Liberati A, Altman DG, Tetzlaff J, Mulrow C, Gotzsche PC, Ioannidis JP, et al. The PRISMA statement for reporting systematic reviews and meta-analyses of studies that evaluate healthcare interventions: explanation and elaboration. BMJ. 2009; 339: b2700.

20. Tierney JF, Stewart LA, Ghersi D, Burdett S, Sydes MR. Practical methods for incorporating summary time-to-event data into meta-analysis. Trials. 2007; 8: 16.

21. Higgins J, Green S. Cochrane handbook of systematic reviews of interventions. New York, USA: Cochrane Collaboration, John Wiley and Sons; 2008.

22. [Internet] Wells GA, Shea B, O'Connell B, Peterson J, Welch V, Losos M, et al. The Newcastle-Ottawa Scale (NOS) for assessing the quality of non-randomised studies in meta-analyses. Accessed 30 June 2016. http://www.ohri.ca/programs/clinical_epidemiology/oxford.asp.

23. [Internet] Phillips B, Ball C, Sackett D. Levels of Evidence (March 2009). Accessed $30 \quad 2016$. http://www.cebm.net/oxford-centre-evidence-based-medicine-levels-eviden ce-march-2009.

24. Parmar MK, Torri V, Stewart L. Extracting summary statistics to perform meta-analyses of the published literature for survival endpoints. Stat Med. 1998; 17: 2815-34.

25. Higgins JP, Thompson SG, Deeks JJ, Altman DG. Measuring inconsistency in meta-analyses. BMJ. 2003; 327: 557-60.

26. Begg CB, Mazumdar M. Operating characteristics of a rank correlation test for publication bias. Biometrics. 1994; 50: 1088-101.

27. Chen KH, Liang ZG, Li L, Qu S, Zeng FY, Pan XB, et al. Comparison of the clinical efficacy of concurrent chemoradiotherapy and radiotherapy alone for 
treating stage II nasopharyngeal carcinoma. [in Chinese]. Chin J Oncol Prev Treat. 2015; 7: 334-9.

28. Chua DT, Ma J, Sham JS, Mai HQ, Choy DT, Hong MH, et al. Improvement of survival after addition of induction chemotherapy to radiotherapy in patients with early-stage nasopharyngeal carcinoma: Subgroup analysis of two Phase III trials. Int J Radiat Oncol Biol Phys. 2006; 65: 1300-6.

29. Lai SZ, Li WF, Chen L, Luo W, Chen YY, Liu LZ, et al. How does intensity-modulated radiotherapy versus conventional two-dimensional radiotherapy influence the treatment results in nasopharyngeal carcinoma patients? Int J Radiat Oncol Biol Phys. 2011; 80: 661-8.

30. Wolden SL, Chen WC, Pfister DG, Kraus DH, Berry SL, Zelefsky MJ. Intensity-modulated radiation therapy (IMRT) for nasopharynx cancer: update of the Memorial Sloan-Kettering experience. Int J Radiat Oncol Biol Phys. 2006; 64: 57-62.

31. Lin S, Lu JJ, Han L, Chen Q, Pan J. Sequential chemotherapy and intensity-modulated radiation therapy in the management of locoregionally advanced nasopharyngeal carcinoma: experience of 370 consecutive cases. BMC cancer. 2010; 10: 39

32. Lee AW, Lau WH, Tung SY, Chua DT, Chappell R, Xu L, et al. Preliminary results of a randomized study on therapeutic gain by concurrent chemotherapy for regionally-advanced nasopharyngeal carcinoma: NPC-9901 Trial by the Hong Kong Nasopharyngeal Cancer Study Group. J Clin Oncol. 2005; 23: 6966-75.

33. Chan AT, Teo PM, Ngan RK, Leung TW, Lau WH, Zee B, et al. Concurrent chemotherapy-radiotherapy compared with radiotherapy alone in locoregionally advanced nasopharyngeal carcinoma: progression-free survival analysis of a phase III randomized trial. J Clin Oncol. 2002; 20: 2038-44.

34. Langendijk JA, Leemans CR, Buter J, Berkhof J, Slotman BJ. The additional value of chemotherapy to radiotherapy in locally advanced nasopharyngeal carcinoma: a meta-analysis of the published literature. J Clin Oncol. 2004; 22: 4604-12.

35. Sun Y, Tang LL, Chen L, Li WF, Mao YP, Liu LZ, et al. Promising treatment outcomes of intensity-modulated radiation therapy for nasopharyngeal carcinoma patients with $\mathrm{N} 0$ disease according to the seventh edition of the AJCC staging system. BMC cancer. 2012; 12: 68.

36. Chen L, Mao YP, Xie FY, Liu LZ, Sun Y, Tian L, et al. The seventh edition of the UICC/AJCC staging system for nasopharyngeal carcinoma is prognostically useful for patients treated with intensity-modulated radiotherapy from an endemic area in China. Radiother Oncol. 2012; 104: 331-7.

37. Xu T, Shen C, Ou X, He X, Ying H, Hu C. The role of adjuvant chemotherapy in nasopharyngeal carcinoma with bulky neck lymph nodes in the era of IMRT. Oncotarget. 2016; 7: 21013-22.

38. Lin JQ, Liang WM, Jan JS, Jiang RS, Lin AC. Another way to estimate outcome of advanced nasopharyngeal carcinoma--is concurrent chemoradiotherapy adequate? Int J Radiat Oncol Biol Phys. 2004; 60: 156-64.

39. Peng H, Chen L, Li WF, Guo R, Zhang Y, Zhang F, et al. Prognostic value of neoadjuvant chemotherapy in locoregionally advanced nasopharyngeal carcinoma with low pre-treatment Epstein-Barr virus DNA: a propensity matched analysis. J Cancer. 2016; 7: 1465-71.

40. Liu N, Chen NY, Cui RX, Li WF, Li Y, Wei RR, et al. Prognostic value of a microRNA signature in nasopharyngeal carcinoma: a microRNA expression analysis. Lancet Oncol. 2012; 13: 633-41.

41. Leung SF, Chan AT, Zee B, Ma B, Chan LY, Johnson PJ, et al. Pretherapy quantitative measurement of circulating Epstein-Barr virus DNA is predictive of posttherapy distant failure in patients with early-stage nasopharyngeal carcinoma of undifferentiated type. Cancer. 2003; 98: 288-91. 